\title{
5-Hydroxymethylation highlights the heterogeneity in keratinization and cell junctions in head and neck cancers
}

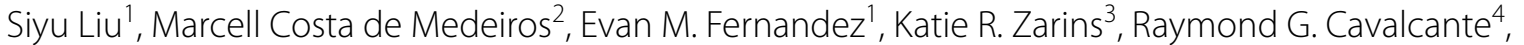 \\ Tingting Qin ${ }^{1}$, Gregory T. Wolf5 ${ }^{5}$ Maria E. Figueroa ${ }^{6}$, Nisha J. D'Silva², Laura S. Rozek ${ }^{3}$ and Maureen A. Sartor, ${ }^{1,7^{*}}$ (D)
}

\begin{abstract}
Background: Head and neck squamous cell carcinoma (HNSCC) is the sixth most prevalent cancer worldwide, with human papillomavirus (HPV)-related HNSCC rising to concerning levels. Extensive clinical, genetic and epigenetic differences exist between HPV-associated HNSCC and HPV-negative HNSCC, which is often linked to tobacco use. However, 5-hydroxymethylation (5hmC), an oxidative derivative of DNA methylation and its heterogeneity among HNSCC subtypes, has not been studied.
\end{abstract}

Results: We characterized genome-wide $5 \mathrm{hmC}$ profiles in HNSCC by HPV status and subtype in $18 \mathrm{HPV}(+)$ and 18 HPV (-) well-characterized tumors. Results showed significant genome-wide hyper-5hmC in HPV(-) tumors, with both promoter and enhancer $5 \mathrm{hmC}$ able to distinguish meaningful tumor subgroups. We identified specific genes whose differential expression by HPV status is driven by differential hydroxymethylation. CDKN2A (p16), used as a key biomarker for HPV status, exhibited the most extensive hyper-5hmC in HPV (+) tumors, while HPV(-) tumors showed hyper-5hmC in CDH13, TIMP2, MMP2 and other cancer-related genes. Among the previously reported two HPV(+) subtypes, IMU (stronger immune response) and KRT (more keratinization), the IMU subtype revealed hyper-5hmC and up-regulation of genes in cell migration, and hypo-5hmC with down-regulation in keratinization and cell junctions. We experimentally validated our key prediction of higher secreted and intracellular protein levels of the invasion gene MMP2 in HPV(-) oral cavity cell lines.

Conclusion: Our results implicate $5 \mathrm{hmC}$ in driving differences in keratinization, cell junctions and other cancerrelated processes among tumor subtypes. We conclude that $5 \mathrm{hmC}$ levels are critical for defining tumor characteristics and potentially used to define clinically meaningful cancer patient subgroups.

Keywords: Head and neck cancer, Human papillomavirus, Hydroxymethylation

\section{Background}

Head and neck squamous cell carcinoma (HNSCC) includes tumors in the oral cavity, larynx and oropharynx, and is the sixth most prevalent cancer worldwide $[1,2]$. Globally, HNSCC affects approximately 680,000

*Correspondence: sartorma@umich.edu

${ }^{1}$ Department of Computational Medicine and Bioinformatics, University of Michigan, 100 Washtenaw Ave., Ann Arbor, MI 48109-2218, USA

Full list of author information is available at the end of the article patients every year, with a five-year survival rate ranging from 37 to $62 \%$ [3]. While tobacco and alcohol consumption are long-recognized risk factors, high-risk strains of human papillomavirus (HPV), in particular HPV-16, account for an increasing number of cases [4]. $\operatorname{HPV}(+)$ HNSCC patients generally show better therapeutic response, improved prognosis and higher overall survival [5-7]. At the molecular level, the gene CDKN2A (p16) is a marker for HPV etiology, due to its high expression level in $\mathrm{HPV}(+)$ tumors and common loss in $\operatorname{HPV}(-)$ 
tumors [8]. A few studies have examined molecular intertumor heterogeneity and identified subtypes of HNSCC $[9,10]$, with the characteristic differences in global gene expression profiles between $\mathrm{HPV}(+)$ and $\mathrm{HPV}(-)$ tumors and among subtypes now established. Results point to differences by HPV status and tumor subtypes in several carcinogenic pathways, including basal epithelial-to-keratinocyte proliferation, immune response, cell adhesion and induction of DNA damage that often correlate with clinical outcome.

Epigenetic differences between normal and HNSCC tumor tissue are extensive, as shown by genome-wide DNA methylation studies [11-13]. $\operatorname{HPV}(+)$ status is associated with hypermethylation in the promoter of several specific genes [14], and HNSCC subtypes have been identified using DNA methylation data from The Cancer Genome Atlas (TCGA) [15]. Previously, we showed that HNSCC DNA methylation profiles correlate with both patient diet and survival $[14,16]$ and extensive genomewide DNA hypomethylation in $\mathrm{HPV}(-)$ compared to $\mathrm{HPV}(+)$ squamous cell carcinoma (SCC) cell lines [17], which has since been validated by others in HNSCC tumors [18].

Most of the above epigenetic data relied on bisulfite treatment of DNA, which does not distinguish between methylation (5-methylcytosine or $5 \mathrm{mC}$ ) and hydroxymethylation (5-hydroxymethylcytosine or $5 \mathrm{hmC}$ ). TET (ten-eleven translocation) proteins can oxidize $5 \mathrm{mC}$ to $5 \mathrm{hmC}$ and other oxidative derivatives, with $5 \mathrm{hmC}$ being the most abundant form in vivo [19-21]. This conversion results in a loss of transcriptional repression in promoters or enhancers, and is a common mechanism to activate genes in differentiation and development [22]. Recent studies found that $5 \mathrm{hmC}$ is depleted in human cancers of many different origins [23-25], yet a recent study of oral cancers found that globally elevated $5 \mathrm{hmC}$ is positively associated with more aggressive tumors and worse survival [26]. Genome-wide 5hmC profiles in HNSCC and in specific tumor subtypes remain uncharacterized, and virtually nothing is known regarding the association of oncogenic viruses such as HPV with $5 \mathrm{hmC}$ levels.

Here, we capture genome-wide hydroxymethylation profiles and examine their heterogeneity among 18 $\mathrm{HPV}(+)$ and $18 \mathrm{HPV}(-)$ previously well-characterized HNSCC tumors. We previously characterized two distinct $\mathrm{HPV}(+) \mathrm{HNSCC}$ subtypes based on gene expression and copy number variation for these 36 tumors and those from TCGA [9]. The IMU subtype is identified by a heightened immune response and more mesenchymal differentiation, whereas the KRT subtype is identified by more keratinization and viral integration events. Based on differential $5 \mathrm{hmC}$ profiles in other human cancers and the fundamental distinctions between $\mathrm{HPV}(+)$ and HPV(-) HNSCC, we reasoned that HPV infection would induce changes in hydroxymethylation, especially near differentiation and developmental genes, and corresponding genes differing by HPV status or tumor subtype. Specifically, since $5 \mathrm{hmC}$ levels are higher in more differentiated cells and lower in stem-like cells [27], we hypothesized an overall higher $5 \mathrm{hmC}$ level in $\mathrm{HPV}(-)$ tumors, since they tend to be more differentiated. Additionally, we predicted differential $5 \mathrm{hmC}$ to exist between the IMU and KRT subtypes.

We used hydroxymethylated DNA immunoprecipitation sequencing (hMeDIP-Seq) to assess $5 \mathrm{hmC}$ in our tumor cohort and integrated results with previously generated RNA-seq data from the same tumors $[28,29]$. Results pointed to extensive differential hydroxymethylation both by HPV status and HPV $(+)$ subtype. The $5 \mathrm{hmC}$ levels at both promoter and enhancer regions distinguish meaningful tumor subgroups and associate with survival. We found a strong positive correlation between hydroxymethylation and gene expression. By integrating $5 \mathrm{hmC}$ with gene expression, we detected important pathways enriched in comparison based on HPV status and subtypes, including keratinization and cell junctions. Finally, we found that a much higher portion of hyperhydroxymethylated regions for $\mathrm{HPV}(-)$ samples fall in keratinocyte enhancer regions compared with $\mathrm{HPV}(+)$ samples. Since some of these enhancers can be linked to differentially expressed target genes, this result indicates that both promoter and enhancer hydroxymethylation play important roles in HNSCC gene regulation. Our results partially explain different mechanisms responsible for previously noted subtype differences and suggest that $5 \mathrm{hmC}$ could be a potential epigenetic target in HNSCC based on HPV status and HPV(+) subtype.

\section{Results}

Widespread differential hydroxymethylation between $\mathrm{HPV}(+)$ and $\mathrm{HPV}(-)$ tumors

The HNSCC cohort consisted of $18 \mathrm{HPV}(+)$ and 18 $\mathrm{HPV}(-)$ patients, as previously determined based on RNA-seq alignment to 14 known high-risk HPV genomes. The cohort consisted of 26 males and 10 females, with an overall median age of 57 years (Additional file 1: Table S1). A total of 14 of the $18 \mathrm{HPV}(+)$ patients were infected by subtype HPV16, and most were former or current smokers. hMeDIP-Seq was performed on these 36 HNSCC tumors to define their genome-wide hydroxymethylation signatures, examine how they differed by HPV status and tumor subtypes, and assess their relationship with clinical variables.

All 36 samples resulted in sufficient quality of data and hundreds of thousands of identified $5 \mathrm{hmC}$ peaks, with the sequencing depth ranging from 67.7 to 152.3 
million reads mapped. The number of peaks detected generally ranged from 208,000 to 480,000 . As expected, the number of peaks was positively correlated with the total number of reads mapped. There were no significant differences between $\mathrm{HPV}(+)$ and $\mathrm{HPV}(-)$ tumors based on peak numbers ( $p$ value $=0.355$, Wilcoxon signed-rank test, Additional file 1: Figure S1A), even after accounting for millions of reads mapped ( $p$ value $=0.287$, ANOVA test).

In general, a much higher level of hydroxymethylation (hyper-5hmC) was reported in HPV(-) HNSCC. Enrichment of $5 \mathrm{hmC}$ levels was plotted over gene bodies, and we observed a consistently higher level of $5 \mathrm{hmC}$ in gene bodies across the genome in $\mathrm{HPV}(-)$ tumors (Fig. 1a). Consistent with previous studies, the average gene body profiles revealed a dip around the transcription start site (TSS) and transcription end site (TES) regions [30].

A total of 19,398 differentially hydroxymethylated regions (DhMRs) were detected as hyper-5hmC in $\mathrm{HPV}(-)$, as opposed to only 2316 in $\mathrm{HPV}(+)$ tumors $\left(p\right.$ value $\left.<10^{-5}\right)$. Most differential peak widths were narrow, between 100 and $200 \mathrm{bp}$, and HPV(-) DhMRs were slightly longer than those for $\mathrm{HPV}(+)$ (Additional file 1: Figure S1B). Although fewer peaks were hyper-5hmC in $\mathrm{HPV}(+)$ samples, they were in general stronger than the HPV(-) DhMRs, with larger fold change (Fig. 1b).

Of the 2316 DhMRs in HPV(+) tumors, about $46 \%$ were annotated to genes, with the majority in introns. Of the 19,398 DhMRs in HPV(-) tumors, more than $72 \%$ were annotated to genes, also with the majority in introns. By comparing the distribution of $\mathrm{HPV}(+)$ and
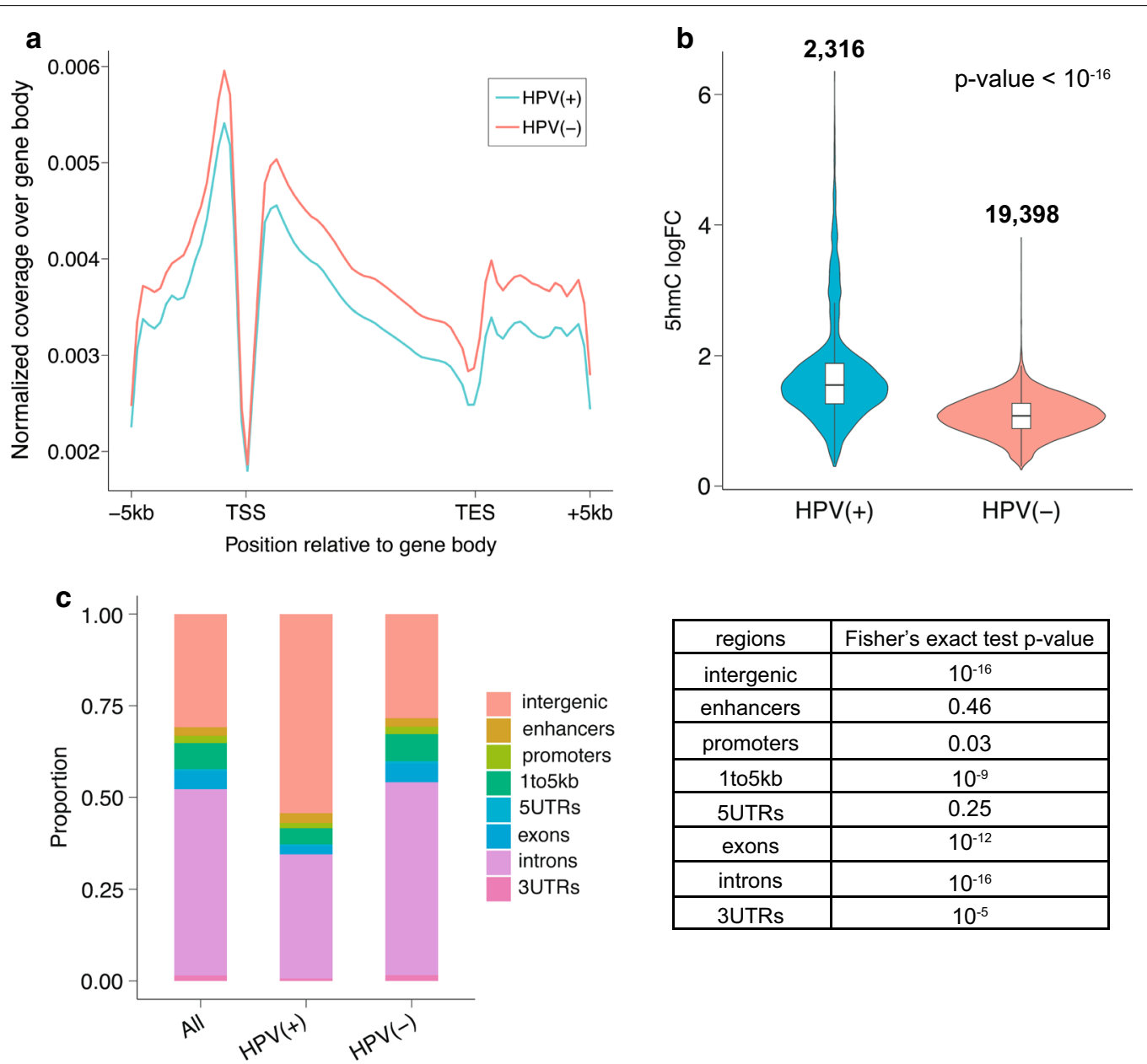

\begin{tabular}{|c|c|}
\hline regions & Fisher's exact test $\mathrm{p}$-value \\
\hline intergenic & $10^{-16}$ \\
\hline enhancers & 0.46 \\
\hline promoters & 0.03 \\
\hline 1to5kb & $10^{-9}$ \\
\hline 5UTRs & 0.25 \\
\hline exons & $10^{-12}$ \\
\hline introns & $10^{-16}$ \\
\hline 3UTRs & $10^{-5}$ \\
\hline
\end{tabular}

Fig. 1 The level and distribution of hydroxymethylation varies by HPV status. a Global 5hmC distribution pattern over gene bodies in both HPV(+) and HPV(-) samples. b Violin plot of $5 \mathrm{hmC} \log \mathrm{FC}$ in HPV (+) tumors (left) and HPV(-) tumors (right). The number on top indicates the total number of peaks being tested. Wilcoxon signed-rank test $p$ value $<10^{-16}$. c The distribution of hyper-5hmC peaks from HPV $(+)$ and HPV $(-)$ samples, where first column represents the combination of $\mathrm{HPV}(+)$ and $\mathrm{HPV}(-)$ tumors. The table on the right displayed the $p$ values from Fisher's exact test between HPV(+) and HPV(-) HNSCC, where exons and introns showed a $p$ value of $10^{-12}$ and $10^{-16}$ respectively 
HPV(-) DhMR annotations to the annotations of random genomic regions, we found a significantly higher proportion of DhMRs were in exons (5.34\%) and introns (52.77\%) in HPV $(-)$ samples, as opposed to a smaller percentage in exons (2.32\%) and introns $(33.98 \%)$ for $\mathrm{HPV}(+)$ DhMRs (Fig. 1c). Together, these results suggest that HPV positivity in HNSCC is linked to a reduced hydroxymethylation signature both in and around genes.

\section{Genes and pathways with hyper-5hmC in $\mathrm{HPV}(+)$ tumors}

A total of 623 genes were associated with hyper-hydroxymethylation in $\mathrm{HPV}(+)$ tumors. CDKN2A (p16), the most important biomarker of HPV status in clinical tests [31], had one of the highest number and also the most significant $\mathrm{HPV}(+)$ DhMRs. In total, 35 genes had hyper- $5 \mathrm{hmC}$ in promoter regions of $\mathrm{HPV}(+)$ tumors. As a prime example, $5 \mathrm{hmC}$ was enriched at the CDKN2A promoter in $\mathrm{HPV}(+)$ cases compared to $\mathrm{HPV}(-)$, a difference that was independent of copy number variations since this is controlled for by the use of input references. This is important, since loss of the CDKN2A locus is known to occur in $\mathrm{HPV}(-)$ cases. The raw coverage depth by sample and peak signal values of CDKN2A both showed great deviation in the promoter and along the gene body between $\mathrm{HPV}(+)$ and $\mathrm{HPV}(-)$ tumors (Additional file 1: Figure S2A). Other important genes found with $\mathrm{HPV}(+)$ DhMRs are listed in Additional file 1: Table S2A.

Pathways enriched with higher $5 \mathrm{hmC}$ in $\mathrm{HPV}(+)$ HNSCC included desmosome, activation of NF-kappaBinducing kinase activity, oxidoreductase activity and mesenchymal cell differentiation $(\mathrm{FDR}<0.1)$ (Additional file 1: Figure S3). Mesenchymal development associated with epithelial to mesenchymal transition (EMT) is consistent with the higher risk of distant metastasis in HPV+ HNSCC. A total of 27 genes displayed higher $5 \mathrm{hmC}$ in $\mathrm{HPV}(+)$ tumors in mesenchymal cell differentiation and development, including the key EMT genes SNAI2, BMP2, SMAD2 and TGFB2, which are part of the TGF $\beta$ / Bone Morphogenic Protein (BMP) signaling pathway [32, 33].

\section{Genes and pathways with hyper-5hmC in HPV(-) tumors}

A larger number of genes, 5584, were found to be hyperhydroxymethylated in $\mathrm{HPV}(-) \mathrm{HNSCC}$, of which 372 genes contained at least one promoter region DhMR. Some of the most important genes with promoter DhMRs were BCAR1, which plays crucial roles in metastasis and cell adhesion, and MMP2, which functions in EMT and immune response in multiple cancer types. We found 204 genes to harbor more than $10 \mathrm{HPV}(-)$ hyper$5 \mathrm{hmC}$ regions. CDH13, a gene encoding a member of the cadherin superfamily that functions in cell-to-cell adhesion and is involved in several diseases, had 83 DhMRs. The peak signal values over each DhMR indeed demonstrated a higher level of hydroxymethylation in $\mathrm{HPV}(-)$ compared with $\mathrm{HPV}(+)$ (Additional file 1: Figure S2B). A more detailed list of important genes with $\mathrm{HPV}(-)$ DhMRs can be found in Additional file 1: Table S2B.

Pathway enrichment results identified cell morphogenesis, cell death, cell motility and cytoskeletal rearrangement/cell-cell junction being among the significantly enriched (FDR <0.1) (Additional file 1: Figure S3). Several previously verified HNSCC-related genes were in a top enriched pathway. For example, frequently mutated HNSCC genes ERBB2, FGD1, NOTCH1, NR4A2, SEMA3E and ARAP3 had HPV(-) DhMRs in cell morphogenesis, and other head and neck-relevant genes such as CTGF, PKN2, TERT, TGFBR2 and TP63 in signal transduction had DhMRs.

\section{Main sources of heterogeneity in hydroxymethylation in promoter and enhancer regions}

We next sought to understand the sources of $5 \mathrm{hmC}$ heterogeneity in our cohort using principle component analysis (PCA). Interestingly, the greatest source of heterogeneity in promoter $5 \mathrm{hmC}$ profiles did not distinguish $\mathrm{HPV}(+)$ from $\mathrm{HPV}(-)$ tumors, but rather one subtype of the HPV $(+)$ tumors (IMU) from all other tumors (see PC2 in Fig. 2a, c). Consistent with the findings in Zhang, et al. [9], the KRT subtype groups closer with $\operatorname{HPV}(-)$ HNSCC, partially due to the shared similarities of heightened keratinization. We thus sought to determine which, if any, known variables could explain the top PCs among $\mathrm{HPV}(+)$ tumors. Correlations between the top principle components and clinical, demographic and batch information were calculated using singular value decomposition (SVD) analysis on proximal promoter regions for the $18 \mathrm{HPV}(+)$ samples. PC1 was correlated with survival ( $p$ value $<0.05)$, while both subtype $(p$ value $<0.01)$ and percentage of epithelial tissue $(p$ value $<0.05)$ were significant in PC2 (Fig. 2e). TILs (tumor-infiltrating lymphocytes) score and batch effect were correlated with PC3, while survival and recurrence $(p$ value $<0.01)$ were correlated with PC4.

In terms of $5 \mathrm{hmC}$ heterogeneity in enhancer regions among the 36 tumors, a similar distinction between IMU and the other tumor samples was observed (see PC2 in Fig. 2b, d). Correlating variables with the top enhancer PCs for the $18 \mathrm{HPV}(+)$ samples, survival was again a significant factor in PC1 ( $p$ value $<0.05)$, and subtype ( $p$ value $<0.01)$ and epithelial tissue $(p$ value $<0.05)$ were again both significant in PC2 (Fig. 2f). However, the separation between the HPV $(+)$ IMU subtype and the rest was not observed for $5 \mathrm{hmC}$ gene body levels (Additional 

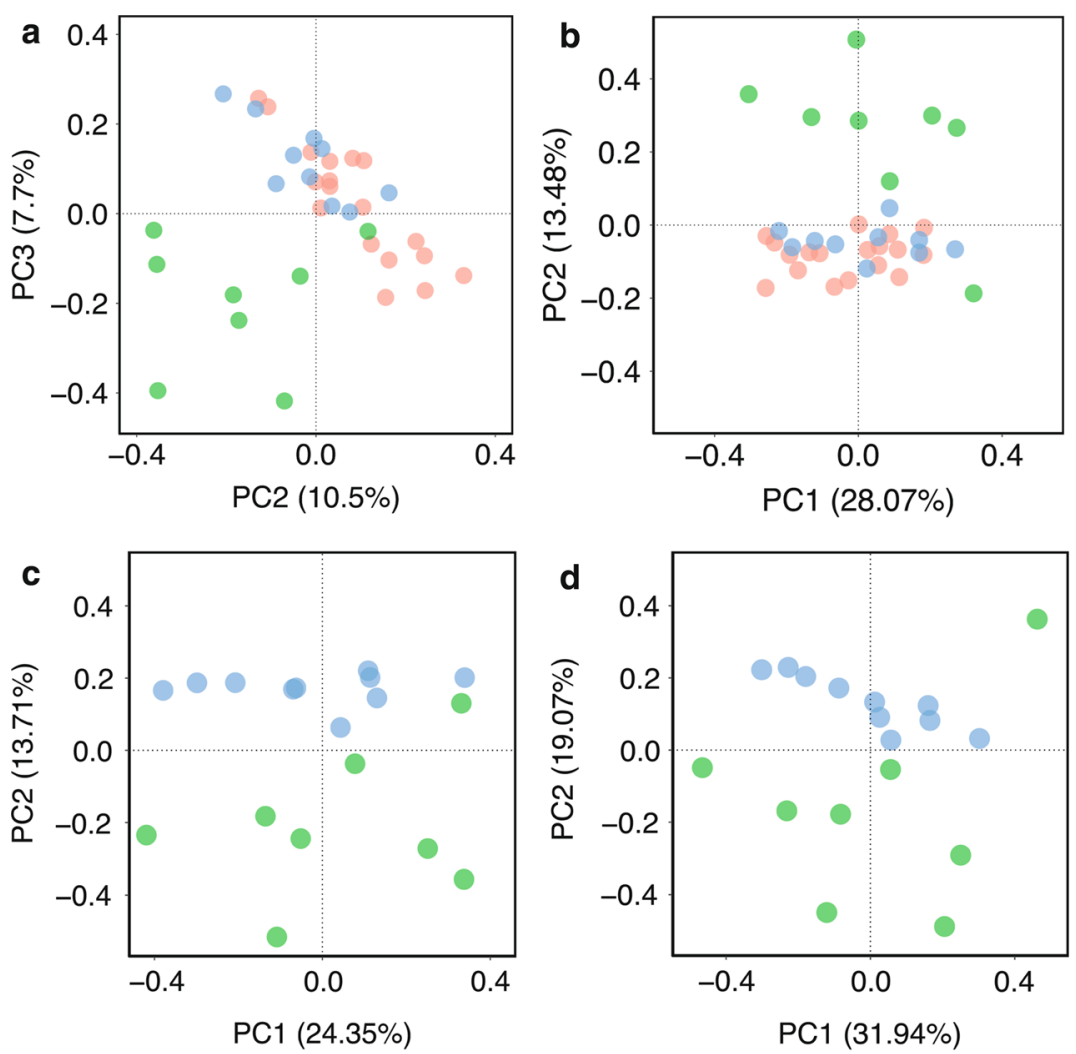

$\operatorname{HPV}(+)$ IMU

$\operatorname{HPV}(+) \mathrm{KRT}$

$\operatorname{HPV}(-)$
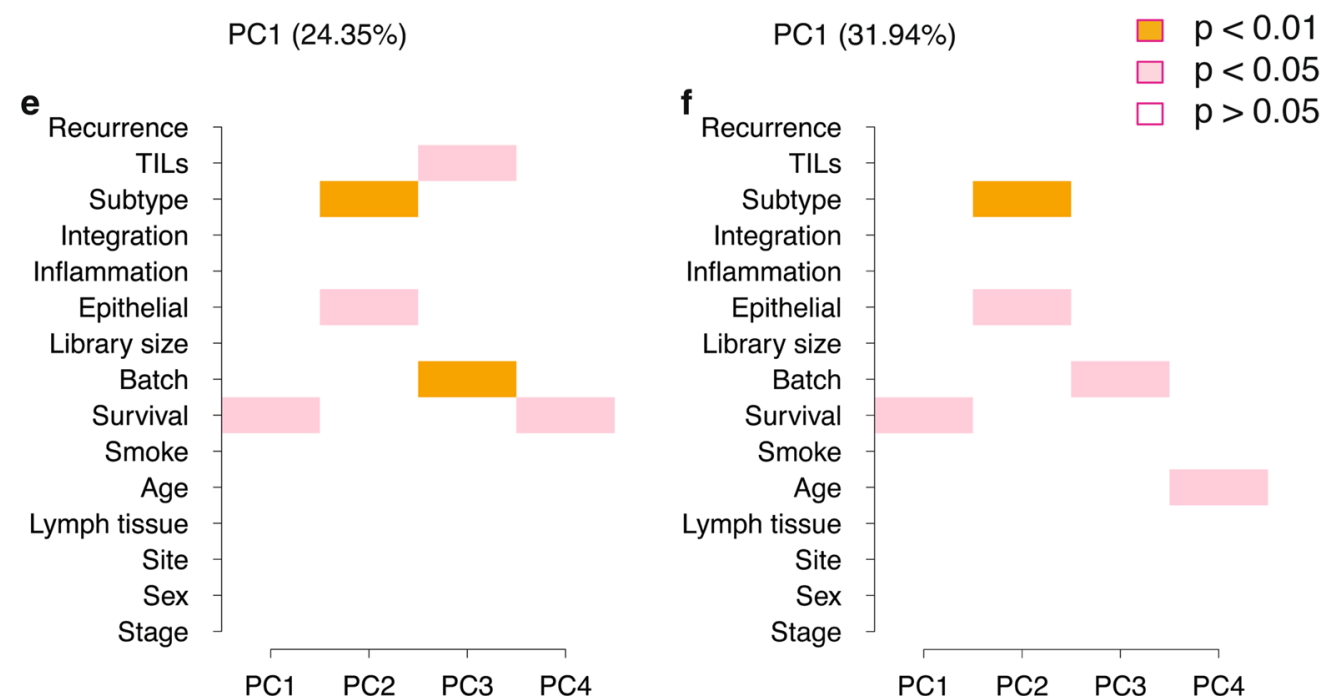

Fig. 2 Principle component analysis illustrated sources of heterogeneity in $5 \mathrm{hmC}$ levels among HPV(+) HNSCC tumors. a PC2 vs PC3 for proximal promoter regions. $\mathbf{b}$ PC1 vs PC2 for custom defined enhancers regions showed clear separation between the IMU subtype and the rest on PC2, which contributed $10.5 \%$ and $13.48 \%$ of the total variance respectively. $\mathbf{c}, \mathbf{d}$ PC1 versus PC2 for both proximal promoters and custom defined enhancers showed clear separation by subtype within HPV (+) tumors. e, $\mathbf{f}$ The SVD analysis on proximal promoters and enhancers demonstrated several relevant clinical variables, such as survival, percentage of epithelial tissue and subtype, which significantly correlated with each principle component in $18 \mathrm{HPV}(+)$ samples

file 1: Figure S4A-D). Instead, SVD analysis on gene body $5 \mathrm{hmC}$ showed other relevant clinical features associated with the top PCs, including survival, lymphocyte tissue and HPV integration status (Additional file 1: Figure S4E-F).

\section{Differential hydroxymethylation and enriched pathways} between the IMU and KRT tumor subtypes

Compared with the IMU subtype, an overall higher level of $5 \mathrm{hmC}$ was observed in the KRT subtype across 
the gene bodies, which was closer to the $5 \mathrm{hmC}$ levels of HPV (-) tumors (Additional file 1: Figure S5A).

In terms of differential $5 \mathrm{hmC}$ between the two $\mathrm{HPV}(+)$ subtypes, there were significantly more instances of hyper-hydroxymethylation in the IMU subtype samples. A total of 63,859 hyper-5hmC regions were found in the IMU subtype, compared with only 1833 hyper$5 \mathrm{hmC}$ regions in the KRT subtype. Only $838(1.3 \%)$ of these regions were also among those found different by HPV status, out of which the majority (771 peaks) were hyperhydroxymethylated in $\mathrm{HPV}(+)$ and IMU tumors (Additional file 1: Figure S5B). Similar to the annotation of DhMRs based on HPV status, the majority of both IMU and KRT DhMRs were mapped to introns (Additional file 1: Figure S5C). A detailed list of important genes with hyper-5hmC in $\mathrm{HPV}(+)$ IMU or KRT tumors can be found in Additional file 1: Table S3A and $\mathrm{S} 3 \mathrm{~B}$, respectively. Interestingly, cancer genes $\mathrm{CDH} 13$ and BCAR1 were found with multiple KRT DhMRs, and they were also important genes for hyper-5hmC in $\mathrm{HPV}(-)$ tumors, which is consistent with the previous finding that the KRT subtype shares more similarities with $\mathrm{HPV}(-)$ HNSCC.

The top enriched pathways marked by hyper-5hmC in the IMU subtype include cornification, epidermis development, keratinocyte differentiation, keratinization and cell differentiation (Additional file 1: Figure S6). For the KRT subtype, cornification and keratinocyte differentiation also appear in the top enriched pathways. However, multiple pathways relevant to cytoskeleton organization or cell-cell junction, including cell adhesion and cytoskeleton structure, were found only in the KRT subtype and were within the top 20 enriched terms (Additional file 1: Figure S6). These terms were also significantly hyper-hydroxymethylated in $\mathrm{HPV}(-)$ compared to $\mathrm{HPV}(+)$, further explaining the similarity of the $\mathrm{HPV}(+) \mathrm{KRT}$ subtype to HPV(-) HNSCC.

\section{Hydroxymethylation is highly associated with gene expression in HNSCCs}

RNA-seq data on the same 36 HNSCC samples were previously analyzed, resulting in 1887 up-regulated and 1644 down-regulated genes in $\mathrm{HPV}(+)$ samples $($ FDR $<0.05$ and absolute fold change $>2)$ [9, 34]. A clear pattern of association can be observed between gene expression and $5 \mathrm{hmC}$ (Pearson's correlation coefficient $=0.62$; odds ratio $(O R)=64.5)$ (Fig. 3a), suggesting that $5 \mathrm{hmC}$ likely drives many of these observed differences. This positive correlation still holds when comparing gene expression with $5 \mathrm{hmC} \operatorname{logFC}$ at enhancer, promoter and gene body separately, with gene body regions showing the strongest correlation (Pearson's correlation coefficient $=0.53$, Additional file 1: Figure S7). The majority of genes (61.4\%) are upregulated and hyper-hydroxymethylated in $\operatorname{HPV}(-)$ tumors, such as cell adhesion genes (including CDH13, CDH11, CDH2, CD44, GLI2, COL4A6), immune response genes (including TGFBR2, CD109, BCAR2, TIMP2, MMP2) and keratinization genes (CDH13, CD109, CDR, PALLD). In particular, TIMP2 and MMP2 also function in tumor invasion.

For CDKN2A, we determined that differential $5 \mathrm{hmC}$ was especially prominent in a $5 \mathrm{~kb}$ region $5^{\prime}$ of the second exon (chr9:21975000-21980000) (Additional file 1: Figure S2A). To assess the association of this particular region with CDKN2A expression, we calculated $5 \mathrm{hmC}$ coverage per sample and found that $5 \mathrm{hmC}$ at this single region explained half of the variability in CDKN2A gene expression levels among the samples (Pearson's correlation coefficient $=0.7$ ). (Fig. 3b).

We next sought to determine the extent to which hydroxymethylation in promoter regions explained differences in gene expression. Twenty genes had at least one hyper-5hmC region in $\mathrm{HPV}(+)$ compared to $\mathrm{HPV}(-)$ in their promoter region, after excluding very low expressed genes. The expression of these genes was indeed significantly higher in HPV(+) IMU than HPV(-) samples (ANOVA $p$ value $=0.00066$ ) (Fig. 3c). The distinction between $\mathrm{HPV}(+)$ and $\mathrm{HPV}(-)$ samples was particularly clear for CDKN2A (Fig. 3d). On the other hand, 296 genes had at least one hyper-5hmC region in $\mathrm{HPV}(-)$ in the promoter. However, there was no significant distinction found in the expression of the top 20 of these genes in any of the three comparisons (Fig. 3c), leading us to hypothesize that the upregulation of genes due to hyper- $5 \mathrm{hmC}$ in $\mathrm{HPV}(-)$ tumors is due to differences at enhancers rather than promoters. By using the genes with hyper- $5 \mathrm{hmC}$ peaks on the promoter regions, we built networks using the shortest paths. The result showed that p16INK4 (CDKN2A) and p14ARF (alternate reading frame protein product of the $\mathrm{CDKN} 2 \mathrm{~A}$ locus) are the two center nodes for $\mathrm{HPV}(+)$ samples (Additional file 1: Figure S8A), while SMAD3, ABCC2 and IL32 are center nodes in the $\mathrm{HPV}(-)$ network (Additional file 1: Figure S8B).

Overall, we identified 35 GO terms both enriched with differential hydroxymethylation (whether proximal to the promoter or distal elements) and differential expression between $\mathrm{HPV}(+)$ and $\mathrm{HPV}(-)$ samples (Fig. 3e). Twenty-five of these $35 \mathrm{GO}$ terms were upregulated and hyper-hydroxymethylated in $\mathrm{HPV}(-)$ samples, including adherens junction, cell morphogenesis, chemotaxis and Ras signaling (Additional file 1: Table S4). This finding suggests that the higher expression of cell junction genes in $\mathrm{HPV}(-)$ tumors is at least partly regulated by hydroxymethylation. It also suggests 

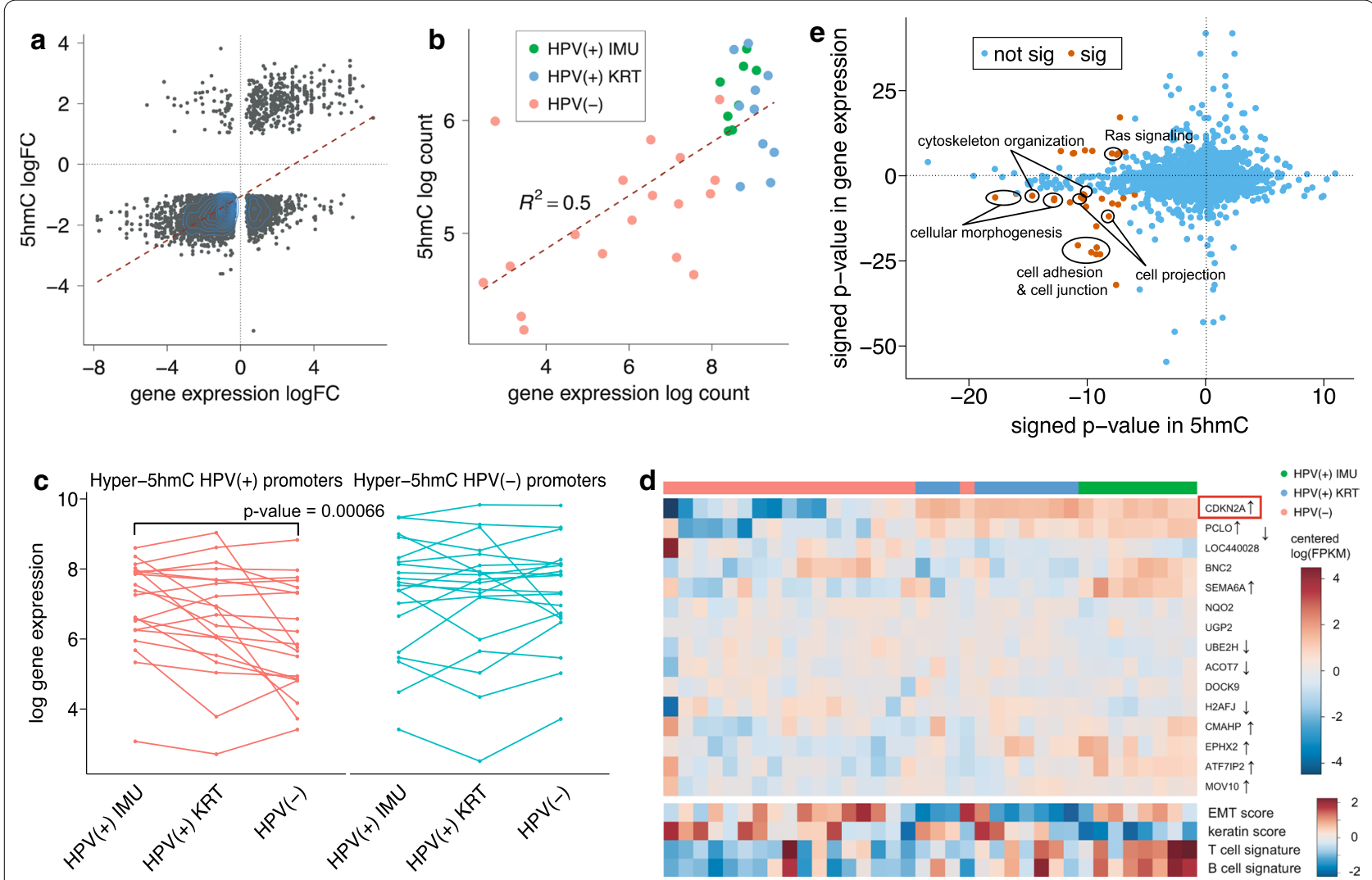

Fig. $35 \mathrm{hmC}$ in HNSCC is highly correlated with gene expression. a Scatterplot showing the positive correlation between gene expression and hydroxymethylation in HPV $(+)$ and HPV $(-)$ samples (Pearson correlation coefficient $=0.62$ ). The top half represents genes that are significantly up-regulated in $\mathrm{HPV}(+)$ tumors, and the right half represents genes that are significantly hyper-hydroxymethylated in HPV(+) tumors. b Scatterplot showing a strong correlation between log gene expression and log 5hmC of 5 kb intron region (chr9:21975000-21980000) of CDKN2A. HPV(+) samples were concentrated near the top right corner, indicating that both their gene expression and $5 \mathrm{hmC}$ coverage were higher compared with HPV (-) samples. c Spaghetti plot of log gene expression for top 20 genes with at least one DhMR in their promoter region for HPV(+) IMU, HPV(+) KRT and HPV(-) samples, respectively. $\mathbf{d}$ Heatmap showing the expression levels of sufficiently expressed genes with at least one $\mathrm{HPV}(+)$ DhMR in their promoter, which were mostly well clustered based on HPV status (marked with black and grey at the top). Most genes were significantly higher expressed in HPV $(+)$ samples, such as CDKN2A. $\uparrow$ indicates genes that are also up-regulated in HPV(+) HNSCC, and $\downarrow$ indicates up-regulation in HPV(-) tumors. Keratin and EMT scores are measurements of keratinization level and EMT level, and T cell signature and B cell signature represent degree of immune response. Generally there was a higher level of keratinization and EMT in HPV(-) samples, while the immune response is more significant in $\mathrm{HPV}(+)$ samples. The detailed calculation can be found in Zhang et al. [9]. e Enrichment analysis results for gene expression vs hydroxymethylation by HPV status. Each dot represents one GO term, and the color denotes the significance (yellow: significant; blue: not significant). Signed $p$ values are defined as $>0$ to indicate up-regulation in HPV(+) samples or hyper-hydroxymethylation in HPV(+) samples, and $<0$ to indicate upregulation in $\mathrm{HPV}(-)$ samples or hyper-hydroxymethylation in $\mathrm{HPV}(-)$ samples

that HPV infection could impact many cell junction biomarkers via the active demethylation process.

Next, we explored the enriched pathways based on $\mathrm{HPV}(+)$ subtype, finding $38 \mathrm{GO}$ terms with significant hyper-5hmC and up-regulation in the IMU subtype, including cell migration, phosphorylation, MAPK cascade and cytokine-mediated signaling pathway (Additional file 1: Figure S5D, Table S5A). Conversely, there were $11 \mathrm{GO}$ terms with significant hyper-5hmC and upregulation in the KRT subtype, including cell-cell junction, keratinization and epidermal cell differentiation, which is consistent with the more differentiated nature of the KRT subtype (Additional file 1: Table S5B).

\section{HPV(-) tumors are more hydroxymethylated in keratinocyte enhancer regions than $\mathrm{HPV}(+)$ tumors}

Since $5 \mathrm{hmC}$ has been shown to be an important mark in enhancer regions [35], we revealed the distribution of hydroxymethylation across samples in relation to different chromatin states, including enhancers, in primary Normal Human Epidermal Keratinocyte (NHEK) cells. NHEK cells are isolated from the epidermis of juvenile 
foreskin or adult skin and are similar to head and neck tissue both morphologically and physiologically [36]. Around the center of NHEK active enhancers and weak enhancers, we observed much higher levels of $5 \mathrm{hmC}$ in $\mathrm{HPV}(-)$ tumors compared with both $\mathrm{HPV}(+)$ subtypes, although all of them showed the expected pattern of an increase around the enhancer centers (Additional file 1: Figure S9). Enhancers also had more differences in hydroxymethylation than promoter regions, as indicated by strikingly more DhMRs in strong enhancers than in active promoters for both $\mathrm{HPV}(+)$ and $\mathrm{HPV}(-)$ tumors (Fig. 4a, Table 1). Fisher's exact test showed an odds ratio (OR) of 2.56 ( $p$ value $<10^{-16}$ ) for strong enhancers and $2.43\left(p\right.$ value $\left.<10^{-16}\right)$ for weak enhancers comparing $\mathrm{HPV}(+)$ vs $\mathrm{HPV}(-)$ samples. This disparity between $\mathrm{HPV}(+)$ and $\operatorname{HPV}(-)$ samples is consistent with the fact

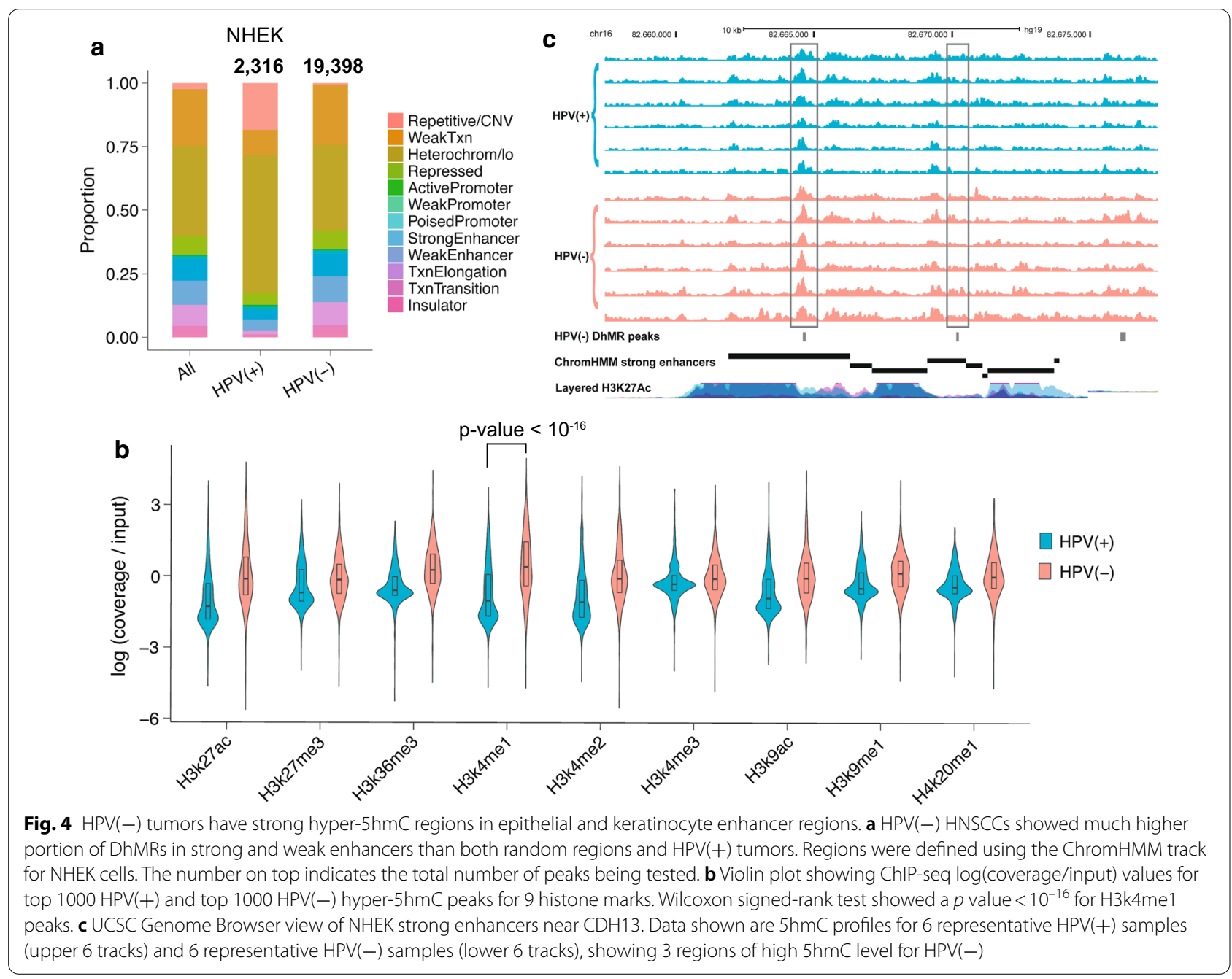

Table 1 Number and percentage of HPV(+), HPV(-), HPV(+) IMU and HPV(+) KRT DhMRs that overlap with keratinocyte enhancers, promoters and super-enhancers

\begin{tabular}{|c|c|c|c|c|c|}
\hline & Strong enhancers & Weak enhancers & Active promoters & $\begin{array}{l}\text { Weak/poised } \\
\text { promoters }\end{array}$ & Super-enhancers \\
\hline HPV(+) DhMRs (2316) & $94 ; 4.06 \%$ & $110 ; 4.75 \%$ & $17 ; 0.73 \%$ & $29 ; 1.25 \%$ & $34 ; 1.47 \%$ \\
\hline HPV(-) DhMRs $(19,398)$ & $1909 ; 9.84 \%$ & $2136 ; 11.01 \%$ & $66 ; 0.34 \%$ & $273 ; 1.41 \%$ & $716 ; 3.45 \%$ \\
\hline HPV(+) IMU DhMRs $(63,859)$ & $4971 ; 7.65 \%$ & $6578 ; 10.30 \%$ & $225 ; 0.35 \%$ & $777 ; 1.22 \%$ & $1374 ; 2.15 \%$ \\
\hline HPV(+) KRT DhMRs (1833) & $230 ; 12.66 \%$ & $197 ; 10.75 \%$ & $8 ; 0.44 \%$ & $20 ; 1.09 \%$ & $117 ; 6.38 \%$ \\
\hline
\end{tabular}


that HPV(-) HNSCCs tend to be more differentiated than $\mathrm{HPV}(+)$ HNSCC.

We reconfirmed the higher keratinocyte (NHEK) enhancer $5 \mathrm{hmC}$ levels in HPV(-) tumors using ChIP-seq data for the histone mark H3K4me1, a mark for active and primed enhancers. Visualizing the H3K4me1 signals for the top 1000 hyper-5hmC regions for HPV(+) and $\mathrm{HPV}(-)$ tumors separately showed the highest signal value within $\mathrm{HPV}(-)$ hyper-5hmC regions (Fig. $4 \mathrm{~b}$ ). Similar to previous findings, this trend was not observed for $\mathrm{H} 3 \mathrm{~K} 4 \mathrm{me} 1$ signals in $\mathrm{HPV}(+)$ tumors, indicating that $\mathrm{HPV}(-)$ samples have higher levels of $5 \mathrm{hmC}$ in keratinocyte enhancer regions.

The target genes of strong enhancers with at least one DhMR were determined using publicly available ChIAPET data (see Additional file 1: Supplementary Methods). In order to study the impact of enhancer hydroxymethylation on target gene expression, we specifically focused on target genes that were also differentially expressed. There were 5 and 66 hyper-hydroxymethylated enhancers associated with differentially up-regulated genes in $\mathrm{HPV}(+)$ and $\mathrm{HPV}(-)$ tumors, respectively. In particular, CLDN1, a cell-to-cell adhesion gene, was the target gene of a $\mathrm{HPV}(+)$ enhancer DhMR and was also up-regulated in $\mathrm{HPV}(+)$ tumors. Conversely, differentially expressed genes CDH13, BCAR1 and TIMP3 not only displayed $\mathrm{HPV}(-)$ enhancer hyper-5hmC, but also contained multiple HPV(-) DhMRs in their exonic and intronic regions. Multiple strong enhancers showed a higher level of hydroxymethylation in $\mathrm{HPV}(-)$ samples in all three of these genes (Fig. 4c).

\section{Expression of invasion gene MMP2 in $\mathrm{HPV}(+)$ and $\mathrm{HPV}(-)$ cell lines}

Our results show that many immune response genes, such as BCAR1, TIMP2 and MMP2, were both higher expressed and hyper-hydroxymethylated in the promoter and sometimes enhancer regions in $\mathrm{HPV}(-)$ tumors. In a previous study, $5 \mathrm{hmC}$ was shown to be positively correlated with depth of tumor invasion in colorectal cancer [37], and depletion of TET1, which could oxidize $5 \mathrm{mC}$ to $5 \mathrm{hmC}$, could facilitate cell invasion [38]. Along these same lines, multiple studies reported that high levels of the MMP2 protein were linked with larger tumor size and more tumor invasion [39]. Therefore, we hypothesized that hyper-hydroxymethylation and higher mRNA levels of MMP2 would result in higher MMP2 secreted protein, which can lead to stronger invasion in $\mathrm{HPV}(-)$ HNSCC.

First we reconfirmed that MMP2 in $\mathrm{HPV}(-)$ tumors showed both higher gene expression and overall higher $5 \mathrm{hmC}$ compared with $\mathrm{HPV}(+)$ tumors, and there is a positive correlation between the gene expression and
5hmC (Fig. 5a). Next, we assessed the secreted and intracellular protein levels of MMP2 in two $\mathrm{HPV}(+)$ oropharynx, one $\mathrm{HPV}(+)$ oral cavity and two $\mathrm{HPV}(-)$ oral cavity cell lines. The zymogram results showed higher levels of secreted MMP2 in HPV(-) cells compared to $\mathrm{HPV}(+)$ oropharynx cell lines, but not $\mathrm{HPV}(+)$ oral cavity (Fig. 5b). Intracellular MMP2 was also higher in the $\mathrm{HPV}(-)$ cell lines than the $\mathrm{HPV}(+)$ oropharynx cells (Fig. 5c). There is no clear distinction in the MMP2 mRNA levels between $\mathrm{HPV}(-)$ oropharynx and $\mathrm{HPV}(+)$ oral cavity cell lines (Fig. 5d).

\section{Discussion}

5-Hydroxymethylcytosine has been shown to be depleted in various human cancers and is known to be more concentrated in differentiated cells [23, 27]. Stem cells, which are closest to the basal epithelial cells in HNSCCs, are known to have lower $5 \mathrm{hmC}$ levels, especially in the gene and enhancer regions required for differentiation $[28,30]$. For our purposes, those differentiation-specific regions would be epithelial and keratinocyte-specific genic and enhancer regions. Differences between $\mathrm{HPV}(+)$ and $\mathrm{HPV}(-)$ HNSCC are extensive in terms of prognosis, tumor recurrence patterns and survival $[5,7,8]$. Similarly, molecular studies have shown marked differences in gene expression, DNA copy numbers and DNA methylation profiles by $\mathrm{HPV}(+)$ status $[9,15,17]$. For instance, multiple studies showed genome-wide DNA hypomethylation in HPV(-) HNSCC tumors [18], and the differential methylation of certain tumor suppressor genes could be potential markers for early HNSCC diagnosis [12].

The HPV lifecycle is tightly linked to epithelial cell differentiation, with HPV initially infecting the undifferentiated basal epithelial cells, and concluding its life cycle in differentiated keratinocytes. Upon HPV oncogene integration, heightened keratinization often occurs, potentially affecting metastatic risk [40]. Our group has shown that patients with integrated HPV E6 \& E7 had significantly worse overall survival [41]. Unlike most cancers, evidence does not suggest that less differentiated HNSCCs are associated with worse survival; indeed, studies have suggested that more differentiated keratinocytes are associated with worse survival in oropharyngeal cancer [42]. Due to the limited sample size of this study, we did not observe any significant survival difference between the two $\mathrm{HPV}(+)$ subtypes, where 2 and 0 deaths were reported after 36 months follow-up in the KRT and IMU subtype, respectively. Another recent publication on meta-analysis of HPV(+) OPSCC followed the observed trend of the IMU subtype having the best survival (labeled $\mathrm{Cl} 1$ in the paper) and the subtype with $100 \%$ HPV integration (similar to our KRT subtype; $\mathrm{Cl} 2$ in paper) having worse survival [43]. 


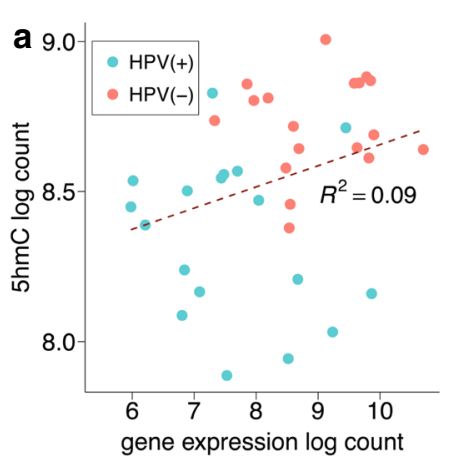

C Immunoblot

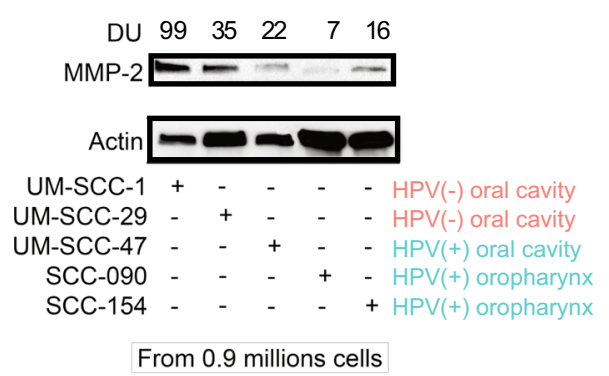

b

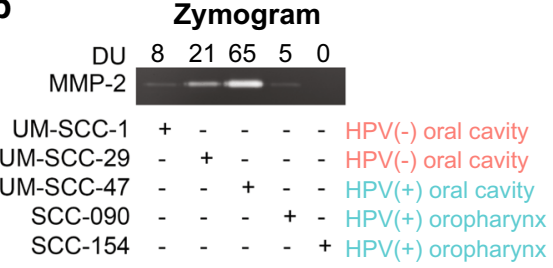

$\mathrm{CM}$ normalized for 1.8 millions cells $/ \mathrm{ml}$

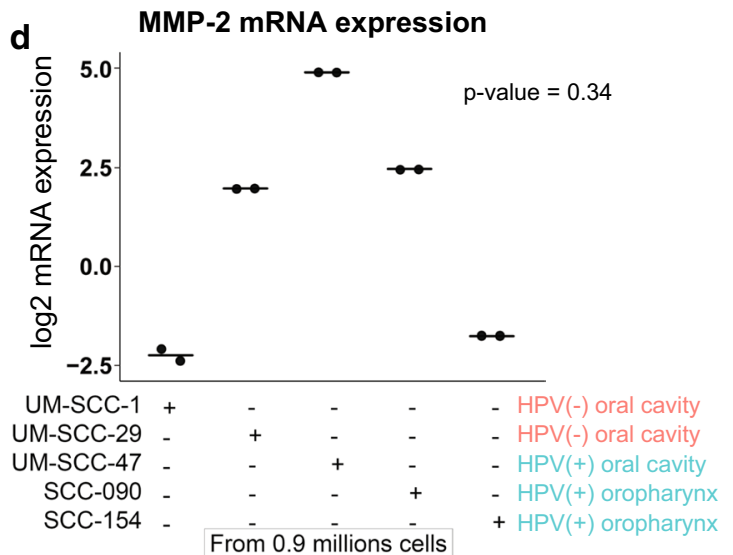

Fig. 5 Protein expression level of MMP2 in $2 \mathrm{HPV}(-)$ and $3 \mathrm{HPV}(+)$ HNSCC cell lines. a Scatterplot showing a correlation between log gene expression and log $5 \mathrm{hmC}$ of MMP2 gene. HPV(-) samples were concentrated near the top right corner, indicating that both their gene expression and $5 \mathrm{hmC}$ coverage were higher compared with HPV $(+)$ samples. b Zymogram and $\mathbf{c}$ immunoblot indicated the secreted and intracellular level of MMP2 protein respectively. $\mathbf{d}$ Dot plot showing the mRNA level of MMP2 with two replicates. After removing the HPV(+) oral cavity cell line, which is an outlier, Wilcoxon signed-rank test showed a $p$ value $=0.34$

Although $5 \mathrm{hmC}$ tends to be overall lower in cancers, others have observed lower $5 \mathrm{hmC}$ in oral cancers to actually be indicative of better prognosis [26] (Fig. 6). This could be due to the confounding effect of HPV; in this study, we found lower $5 \mathrm{hmC}$ in $\mathrm{HPV}(+)$ patients, who have less differentiated tumors and better prognosis (Fig. 6). However, mesenchymal differentiation may also lead to loco-regional or distant metastasis; thus, the complete relationship between differentiation and metastasis appears to be complex, as suggested by studies in oral cancer [44]. Among our differential $5 \mathrm{hmC}$ genes, only CDK6 was identified as a clinically actionable target. CDK6 was hyper-hydroxymethylated in $\mathrm{HPV}(-)$ tumors at seven intronic regions, is targeted in the treatment of certain breast cancers [45] and recently showed response in the treatment of oral squamous cell carcinoma [46]. $\mathrm{CD} 20$, which is regulated by the epigenetic markers $\mathrm{NF \kappa B}$ and SMAD2/3 [47], is targeted in chronic lymphocytic leukemia and follicular lymphoma [48]. The NFKB pathway and SMAD2/3 genes were identified as important $5 \mathrm{hmC}$ markers in our study, suggesting a potential use of B cell markers in the immunotherapy of HNSCC.

Our study is the first to characterize genome-wide DNA hydroxymethylation in head and neck cancers.
Among differentiation genes, we found strong hyper$5 \mathrm{hmC}$ in $\mathrm{HPV}(-)$ tumors especially concentrated in cell junction and cell adhesion pathways, which is consistent with previous findings that $\mathrm{HPV}(-) \mathrm{HNSCC}$ is more differentiated compared with HPV(+) HNSCC. The overall higher level of hydroxymethylation in HPV(-) HNSCCs is also consistent with observed overall higher levels of DNA methylation in $\mathrm{HPV}(+)$ oropharyngeal cancer cases, considering the antagonizing effect of methylation and hydroxymethylation [18]. Similar to DNA methylation, profiles of hydroxymethylation were highly affected by HPV status, particularly for p16. The great majority of hyper-hydroxymethylated genes were in $\operatorname{HPV}(-)$ HNSCC, many of which play important roles in cancer pathways. CDH13, a cell-cell adhesion gene and a member of the cadherin superfamily, was upregulated in $\mathrm{HPV}(-)$ tumors and also had the highest number of hyper-5hmC regions in $\mathrm{HPV}(-)$. Interestingly, other major cadherin family genes (e.g., $\mathrm{CDH} 1$ and $\mathrm{CDH} 11$ ) are hypermethylated in $\operatorname{HPV}(+)$ HNSCC $[17,49]$. Some genes known to have differential methylation by HPV status also displayed differential $5 \mathrm{hmC}$ in the opposite direction, including cell adhesion genes COL4A6 and BCAR1, and tumor suppressor genes TIMP3 and SFRP4. 


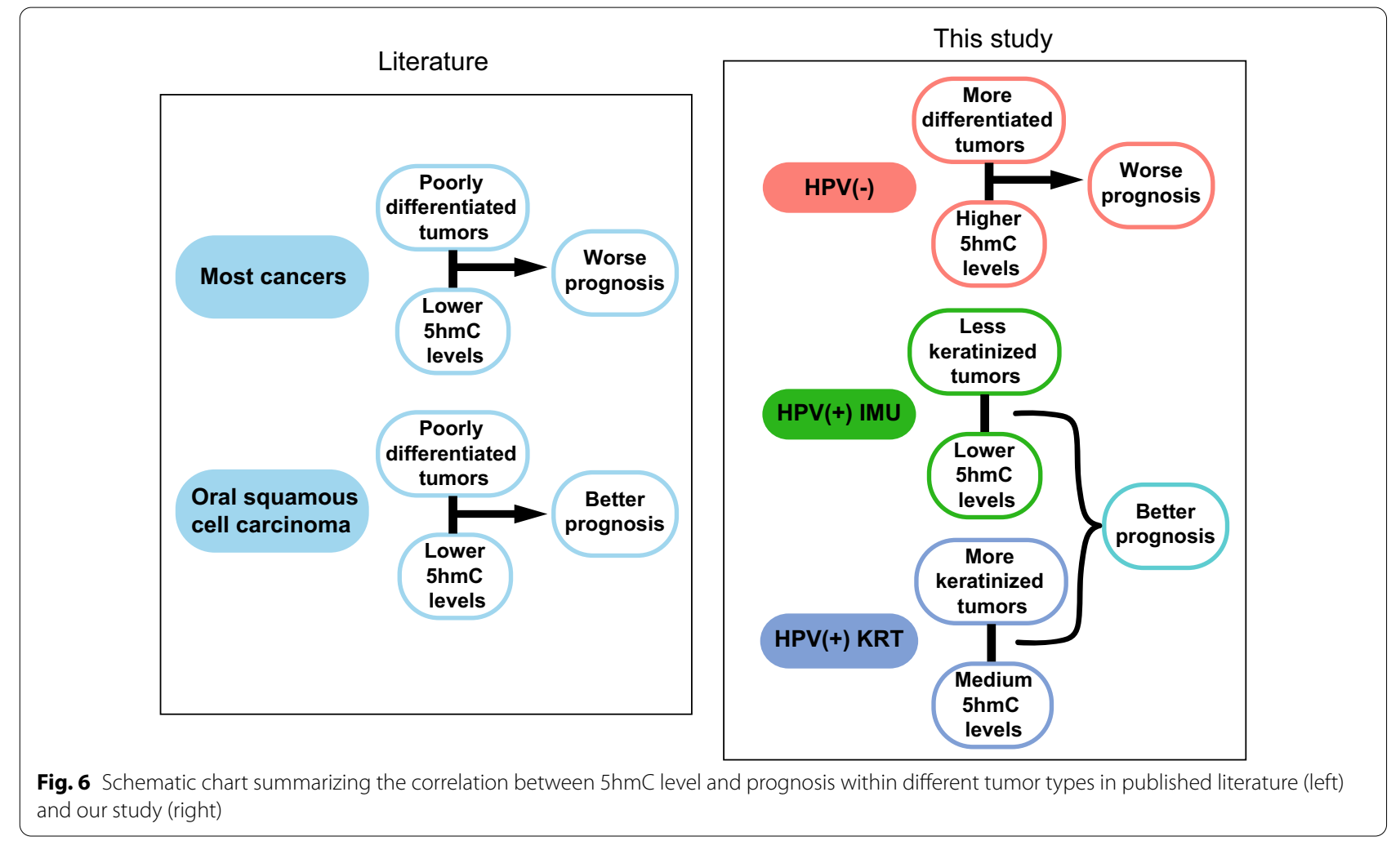

Even more than distinguishing HNSCC tumors by HPV status, $5 \mathrm{hmC}$ profiles distinguished the IMU HPV $(+)$ subtype from the KRT HPV $(+)$ subtype and $\mathrm{HPV}(-)$ tumors. The KRT subtype is more similar to $\mathrm{HPV}(-)$ HNSCC than the IMU subtype based on gene expression [9]. Consistent with this, we found the same based on $5 \mathrm{hmC}$, with a much higher level of $5 \mathrm{hmC}$ found in the KRT subtype, which is furthermore consistent with the more differentiated nature of this subtype. We also found cancer pathways such as cell migration enriched with hyper-5hmC in the IMU subtype, while cornification and keratinization were significantly enriched with hyper$5 \mathrm{hmC}$ in the KRT subtype.

A recent study on hydroxymethylation of pancreatic cancer showed positive correlation between $5 \mathrm{hmC}$ and open chromatin generated ATAC-seq in both cancer and control cells [50]. While we did not have ATAC-seq data available, in our study we found a similar correlation with ChromHMM tracks of NHEK cells [51], and the especially strong enrichment of $5 \mathrm{hmC}$ on enhancers in $\mathrm{HPV}(-) \mathrm{HNSCC}$ could partially be attributed to its more differentiated and/or malignant nature.

In summary, our comprehensive characterization of the genome-wide hydroxymethylation profiles in HNSCC revealed significant differential hydroxymethylation both by HPV status and between $\mathrm{HPV}(+)$ subtypes. We report the significance of CDKN2A hydroxymethylation by
HPV status, as well as many other cancer-related genes, such as CDH1, TIMP3 and SFRP4. Overall, the results are closely in line with current knowledge of differences by HPV status, including differences in DNA methylation. We also discovered the important role of the less reported gene CDH13 in HNSCC, and that the differential hydroxymethylation was especially concentrated in CDH13 enhancer regions.

\section{Conclusions}

In conclusion, although $5 \mathrm{hmC}$ marks genes across a wide array of cellular processes, $5 \mathrm{hmC}$ profiles highlight genes turned on during differentiation, and can therefore be used for an in-depth characterization of the differentiation state of tumors. Thus, genome-wide $5 \mathrm{hmC}$ analysis is beneficial, especially to the extent that differentiation state affects carcinogenic pathways, including cell junctions and adhesion, invasion and migration in our study. Our data suggest that $5 \mathrm{hmC}$ profile for the IMU subtype could be a useful biomarker in HPV positive cancers and should be explored further.

As the first study to characterize the genome-wide $5 \mathrm{hmC}$ profile in HNSCC, we identified significant genome-wide hyper-5hmC in $\mathrm{HPV}(-)$ tumors, with both promoter and enhancer $5 \mathrm{hmC}$ levels being clinically relevant and able to distinguish meaningful tumor subgroups. We also implicated $5 \mathrm{hmC}$ in key cancer-related 
processes that determine the likelihood of metastasis in head and neck cancer. genes. Clinically, therapeutic deescalation schedules are being introduced for $\mathrm{HPV}(+)$ patients, but the current challenge to such changes includes better identification of the small subset of $\mathrm{HPV}+$ cancer patients that have poor prognosis. Our study has important implications that $5 \mathrm{hmC}$ levels are crucial in defining tumor characteristics and potentially used to define clinically meaningful cancer patient subgroups for many cancer types.

\section{Methods}

\section{Patient recruitment and hMeDIP-seq protocol}

From 2011 to 2013, we identified 36 incident HNSCC patients with pre-treatment oropharynx or oral cavity squamous cell carcinoma at Michigan Medicine Hospital. HPV status was determined based on p16 staining and RNA-seq, as previously described [9, 41]. The details of tumor tissue acquisition can be found in Additional file 1: Supplementary Methods. After DNA extraction, the quality of the 36 DNA samples was measured by TapeStation genomic DNA kit (Agilent, Santa Clara, CA), followed by quantitation assessment by Qubit broad range dsDNA (ThermoFisher, Carlsbad, CA). Enzymes, PCR primers and indexed adaptors were supplied by New England BioLabs (Ipswich, MA) and Integrated DNA Technologies (Coralville, CA), respectively.

A total of $1 \mu \mathrm{g}$ of genomic DNA was used for shearing, blunt-end repair and phosphorylation process, and a single adenine nucleotide was then added to the $3^{\prime}$ end of the resulting fragments for ligation preparation. DNA was cleaned by Qiagen's MinElute PCR purification columns (Qiagen, Germantown, MD). DNA samples were denatured and resuspended in ice-cold immunoprecipitation buffer after the addition of DNA spike-ins for hMeDIP (Diagenode Denville, NJ). At this stage, 10\% volume of the DNA solutions were kept as inputs, and immunoprecipitation overnight at $4{ }^{\circ} \mathrm{C}$ with rotation was performed on the remaining solution, after adding a $5 \mathrm{hmC}$-specific antibody (Cat \# 39791, Active Motif, Carlsbad, CA). The $5 \mathrm{hmC}$-enriched DNA fragments (IP) were released from the antibody and cleaned-up by Proteinase $\mathrm{K}$ (ThermoFisher, Carlsbad, CA) and AMPure XP beads (Beckman Coulter, Brea, $\mathrm{CA}$ ), respectively. In order to evaluate the percent enrichment over input in the IP, qPCR with primers for spike-ins was conducted. For samples with good percent enrichment over input, PCR amplification was performed for library production, followed by cleaning with AMPure XP beads and quantification with the Qubit assay (ThermoFisher, Carlsbad, CA) and TapeStation High Sensitivity D1000 kit (Agilent, Santa Clara, CA). Each hMeDIP-seq sample with paired input was sequenced on a single lane of an Illumina HiSeq 2500, generating single-end, $50 \mathrm{bp}$ reads.

\section{hMeDIP-seq data analysis and peak finding}

The main analysis steps were conducted using the Methylation Integration (mint) pipeline [52]. Sample quality was first assessed with FastQC [53], and then, reads were aligned with bowtie2 [54] after adapter and quality trimming with Trim Galore! Peaks for each sample compared to input, i.e. the genome-wide regions of hydroxymethylation, were identified using MACS2.

Differential peaks, i.e. differentially hydroxymethylated regions (DhMRs) between $\operatorname{HPV}(+)$ and $\operatorname{HPV}(-)$ samples, or between $\mathrm{HPV}(+)$ subtypes, were identified using PePr [55]. PePr takes replicates into account using a negative binomial model while improving variability estimates using information from neighboring sites. Differential peaks were called with false discovery rate (FDR) $<0.05$ and fold change $(\mathrm{FC})>2$. Peaks were annotated using the R Bioconductor package annotatr [56]. Peaks annotated to $\mathrm{X}$ and $\mathrm{Y}$ chromosomes were excluded to avoid confounding by sex. The overall $5 \mathrm{hmC}$ levels over gene bodies were calculated using MACS2 peaks with metaGeneProfile function in HOMER [57].

\section{Principle component analysis (PCA) and singular value decomposition (SVD) analysis}

PCA was performed using prcomp function in $\mathrm{R}$, with the use of hMeDIP-seq counts in proximal promoters, gene bodies and custom enhancer regions. $\mathrm{X}$ and $\mathrm{Y}$ chromosome reads were removed to avoid sex bias. The bedtools intersect function was used to obtain $5 \mathrm{hmC}$ counts in promoter regions ( $1 \mathrm{~kb}$ before to $1 \mathrm{~kb}$ after TSS's) and gene bodies (from TSS to TES), followed by normalization by manual specification of library sizes in DESeq2, with the input values as covariate. The background was taken into account by calculating the log2 fold change for each region. SVD analysis was performed on the top principal components using the Bioconductor package ChAMP [58], to study correlation with variables of interest.

\section{Additional analyses}

Detailed analysis methods for (1) generation of custom enhancer definitions; (2) RNA-seq analyses and association with $5 \mathrm{hmC}$; (3) gene set enrichment testing on hMeDIP-seq and RNA-seq data; (4) keratinocyte enhancer regions download and analysis; (5) experimental validation with $\mathrm{HPV}(+)$ and $\mathrm{HPV}(-)$ cell lines are available in the Additional file 1: Supplementary Methods. 


\section{Supplementary information}

Supplementary information accompanies this paper at https://doi. org/10.1186/s13148-020-00965-8.

Additional file 1. Supplementary materials: methods, figures and tables.

\section{Abbreviations}

5mC: 5-Methylcytosine; 5hmC: 5-Hydroxymethylcytosine; BMP: Bone morphogenic protein; ANOVA: Analysis of variance; DhMRs: Differentially hydroxymethylated regions; EMT: Epithelial to mesenchymal transition; FDR: False discovery rate; GO: Gene ontology; hMeDIP-Seq: Hydroxymethylated DNA immunoprecipitation sequencing; HNSCC: Head and neck squamous cell carcinoma; HPV: Human papillomavirus; IMU: Immune response; KRT: Keratinization; NHEK: Normal human epidermal keratinocyte; OR: Odds ratio; PC: Principle component; PCA: Principle component analysis; SCC: Squamous cell carcinoma; TCGA: The Cancer Genome Atlas; TET: Ten-eleven translocation; TES: Transcription end site; TGFB: Transforming growth factor beta; TSS: Transcription start site; SVD: Singular value decomposition.

\section{Acknowledgements}

We thank the University of Michigan Epigenomics Core for their careful sample and library preparations. Dr. Maria Figueroa was supported by a Leukemia and Lymphoma Society Scholar award.

\section{Authors' contributions}

S.L. performed the bioinformatics analyses, interpreted the sequencing data and prepared the manuscript; M.C.M. conducted the molecular biology experiments; E.F. and R.G.C contributed in additional bioinformatics analyses; K.R.Z collected and prepared both clinical data and tumor tissues in the $36 \mathrm{UM}$ cohort; T.Q., M.E.F. and G.T.W. helped improve the manuscript; N.J.D. designed and supervised the molecular biology experiments; M.A.S. and M.E.F. had the initial idea for the study and secured funding, while M.A.S and L.S.R. supervised the bioinformatics analyses, data interpretation and manuscript preparation. All authors read and approved the final manuscript.

\section{Funding}

This work was supported by funding from the National Cancer Institute, National Institutes of Health Grants [R21-CA187537], [R01-CA158286], [R01-CA250214], and the University of Michigan Rogel Cancer Center Grant [P30-CA046592] and University of Michigan Specialized Programs of Research Excellence (SPORE) Grant [P50-CA097248]. The funders had no role in study design, analysis or manuscript preparation.

\section{Availability of data and materials}

Raw hMeDIP-seq data and MACS2 peaks supporting the conclusions of this article have been deposited in NCBI's Gene Expression Omnibus and can be accessed with accession number GSE144034. RNA-seq data is available at GSE74956.

\section{Ethics approval and consent to participate}

The study was approved by the University of Michigan Institutional Review Board. All patients have given written consent for the study. The experimental methods comply with the Helsinki Declaration.

\section{Consent for publication}

Not applicable.

\section{Competing interests}

The authors declare that they have no competing interests.

\section{Author details}

${ }^{1}$ Department of Computational Medicine and Bioinformatics, University of Michigan, 100 Washtenaw Ave., Ann Arbor, MI 48109-2218, USA. ${ }^{2}$ Department of Periodontics and Oral Medicine, University of Michigan, Ann Arbor, MI 48104, USA. ${ }^{3}$ Department of Environmental Health Sciences, University of Michigan, Ann Arbor, MI 48109, USA. ${ }^{4}$ Epigenomics Core, University of Michigan, Ann Arbor, Ml 48109, USA. ${ }^{5}$ Department of Otolaryngology-Head and Neck Surgery, Michigan Medicine, Ann Arbor, MI 48109, USA.
${ }^{6}$ Department of Human Genetics and Sylvester Comprehensive Cancer Center, Miller School of Medicine, University of Miami, Miami, FL 33136, USA. ${ }^{7}$ Department of Biostatistics, University of Michigan, Ann Arbor, MI 48109, USA.

Received: 10 June 2020 Accepted: 3 November 2020

Published online: 17 November 2020

\section{References}

1. Mendenhall WM, Mancuso AA, Amdur RJ, Stringer SP, Villaret DB, Cassisi NJ. Squamous cell carcinoma metastatic to the neck from an unknown head and neck primary site. Am J Otolaryngol. 2001;22(4):261-7.

2. Argiris A, Karamouzis MV, Raben D, Ferris RL. Head and neck cancer. The Lancet. 2008;271:1695-709.

3. Ferlay J, Colombet M, Soerjomataram I, Mathers C, Parkin DM, Piñeros $M$, et al. Estimating the global cancer incidence and mortality in 2018 GLOBOCAN sources and methods. Int J Cancer. 2019;144:1941-53.

4. Chaturvedi AK, Engels EA, Pfeiffer RM, Hernandez BY, Xiao W, Kim E, et al. Human papillomavirus and rising oropharyngeal cancer incidence in the United States. J Clin Oncol. 2011;29:4294.

5. Blitzer GC, Smith MA, Harris SL, Kimple RJ. Review of the clinical and biologic aspects of human papillomavirus-positive squamous cell carcinomas of the head and neck. Int J Radiat Oncol Biol Phys. 2014;88:761-70.

6. Ang KK, Harris J, Wheeler R, Weber R, Rosenthal DI, Nguyen-Tân PF, et al. Human papillomavirus and survival of patients with oropharyngeal cancer. N Engl J Med. 2010;363:24-35.

7. Dok R, Nuyts S. HPV positive head and neck cancers: molecular pathogenesis and evolving treatment strategies. Cancers. 2016;8:41.

8. Chung $\mathrm{CH}$, Zhang Q, Kong CS, Harris J, Fertig EJ, Harari PM, et al. p16 protein expression and human papillomavirus status as prognostic biomarkers of nonoropharyngeal head and neck squamous cell carcinoma. J Clin Oncol. 2014;32(35):3930-8.

9. Zhang Y, Koneva LA, Virani S, Arthur AE, Virani A, Hall PB, et al. Subtypes of HPV-positive head and neck cancers are associated with HPV characteristics, copy number alterations, PIK3CA mutation, and pathway signatures. Clin Cancer Res. 2016;22(18):4735-45.

10. Papillon-Cavanagh S, Lu C, Gayden T, Mikael LG, Bechet D, Karamboulas C, et al. Impaired H3K36 methylation defines a subset of head and neck squamous cell carcinomas. Nat Genet. 2017;49:180-5.

11. Baba S, Yamada Y, Hatano Y, Miyazaki Y, Mori H, Shibata T, et al. Global DNA hypomethylation suppresses squamous carcinogenesis in the tongue and esophagus. Cancer Sci. 2009;100:1186-91.

12. Lim Y, Sun CX, Tran P, Punyadeera C. Salivary epigenetic biomarkers in head and neck squamous cell carcinomas. Biomarkers in Medicine. 2016;10:301-13.

13. Castilho RM, Squarize $\mathrm{CH}$, Almeida LO. Epigenetic modifications and head and neck cancer: implications for tumor progression and resistance to therapy. Int J Mol Sci. 2017;18(7):1506.

14. DegliEsposti D, Sklias A, Lima SC, Beghelli-de la Forest Divonne S, Cahais $\checkmark$, Fernandez-Jimenez N, et al. Unique DNA methylation signature in HPV-positive head and neck squamous cell carcinomas. Genome Med. 2017:9:1-12.

15. Lawrence MS, Sougnez C, Lichtenstein L, Cibulskis K, Lander E, Gabriel $\mathrm{SB}$, et al. Comprehensive genomic characterization of head and neck squamous cell carcinomas. Nature. 2015;517:576-82.

16. Choudhury JH, Ghosh SK. Promoter hypermethylation profiling identifies subtypes of head and neck cancer with distinct viral, environmental, genetic and survival characteristics. PLOS ONE. 2015;10(6):e0129808.

17. Sartor MA, Dolinoy DC, Jones TR, Colacino JA, Prince MEP, Carey TE, et al. Genome-wide methylation and expression differences in HPV $(+)$ and $\mathrm{HPV}(-)$ squamous cell carcinoma cell lines are consistent with divergent mechanisms of carcinogenesis. Epigenetics. 2011;6(6):777-87.

18. Lleras RA, Smith RV, Adrien LR, Schlecht NF, Burk RD, Harris TM, et al. Unique DNA methylation loci distinguish anatomic site and HPV status in head and neck squamous cell carcinoma. Clin Cancer Res. 2013;19(19):5444-55. 
19. Tahiliani M, Koh KP, Shen Y, Pastor WA, Bandukwala H, Brudno Y, et al. Conversion of 5-methylcytosine to 5-hydroxymethylcytosine in mammalian DNA by MLL partner TET1. Science (80-). 2009;324(5929):930-5.

20. Ruzov A, Tsenkina Y, Serio A, Dudnakova T, Fletcher J, Bai Y, et al. Lineagespecific distribution of high levels of genomic 5-hydroxymethylcytosine in mammalian development. Cell Res. 2011;21(9):1332-42.

21. Tan L, Xiong L, Xu W, Wu F, Huang N, Xu Y, et al. Genome-wide comparison of DNA hydroxymethylation in mouse embryonic stem cells and neural progenitor cells by a new comparative hMeDIP-seq method. Nucleic Acids Res. 2013;41:e84.

22. Laird A, Thomson JP, Harrison DJ, Meehan RR. 5-hydroxymethylcytosine profiling as an indicator of cellular state. Epigenomics. 2013;5:655-69.

23. Jin SG, Jiang Y, Qiu R, Rauch TA, Wang Y, Schackert G, et al. 5-hydroxymethylcytosine is strongly depleted in human cancers but its levels do not correlate with IDH1 mutations. Cancer Res. 2011;71(24):7360-5.

24. Yang H, Liu Y, Bai F, Zhang JY, Ma SH, Liu J, et al. Tumor development is associated with decrease of TET gene expression and 5-methylcytosine hydroxylation. Oncogene. 2013;32(5):663-9.

25. Jäwert F, Hasséus B, Kjeller G, Magnusson B, Sand L, Larsson L. Loss of 5-hydroxymethylcytosine and TET2 in oral squamous cell carcinoma. Anticancer Res. 2013;33(10):4325-8.

26. Wang Y, Hu H, Wang Q, Li Z, Zhu Y, Zhang W, et al. The level and clinical significance of 5-hydroxymethylcytosine in oral squamous cell carcinoma: An immunohistochemical study in 95 patients. Pathol Res Pract. 2017;213:969-74.

27. Ficz G, Gribben JG. Loss of 5-hydroxymethylcytosine in cancer: cause or consequence? Genomics. 2014;104:352-7.

28. Ficz G, Branco MR, Seisenberger S, Santos F, Krueger F, Hore TA, et al. Dynamic regulation of 5-hydroxymethylcytosine in mouse ES cells and during differentiation. Nature. 2011;473(7347):398-404.

29. Wu H, D'Alessio AC, Ito S, Wang Z, Cui K, Zhao K, et al. Genome-wide analysis of 5-hydroxymethylcytosine distribution reveals its dual function in transcriptional regulation in mouse embryonic stem cells. Genes Dev. 2011;25:679-84

30. Wernig-Zorc S, Yadav MP, Kopparapu PK, Bemark M, Kristjansdottir HL, Andersson PO, et al. Global distribution of DNA hydroxymethylation and DNA methylation in chronic lymphocytic leukemia. Epigenetics Chromatin. 2019:12:1-5.

31. Li J, Poi MJ, Tsai MD. Regulatory mechanisms of tumor suppressor P16INK4A and their relevance to cancer. Biochemistry. 2011;50:5566-82.

32. Fenouille N, Tichet M, Dufies M, Pottier A, Mogha A, Soo JK, et al. The epithelial-mesenchymal transition (EMT) regulatory factor SLUG (SNAI2) is a downstream target of SPARC and AKT in promoting melanoma cell invasion. PLoS ONE. 2012;7(7):e40378.

33. Rahman MS, Akhtar N, Jamil HM, Banik RS, Asaduzzaman SM. TGF-B/BMP signaling and other molecular events: regulation of osteoblastogenesis and bone formation. Bone Research. 2015;3:15005.

34. Robinson MD, McCarthy DJ, Smyth GK. edgeR: a bioconductor package for differential expression analysis of digital gene expression data. Bioinformatics. 2009;26:139-40

35. Li J, Wu X, Zhou Y, Lee M, Guo L, Han W, et al. Decoding the dynamic DNA methylation and hydroxymethylation landscapes in endodermal lineage intermediates during pancreatic differentiation of hESC. Nucleic Acids Res. 2018;46:2883-900.

36. Cohen EEW, Lingen MW, Zhu B, Zhu H, Straza MW, Pierce C, et al. Protein kinase $C \zeta$ mediates epidermal growth factor-induced growth of head and neck tumor cells by regulating mitogen-activated protein kinase. Cancer Res. 2006;66:6296-303.

37. Tian Y, Lin A, Gan M, Wang H, Yu D, Lai C, et al. Global changes of 5-hydroxymethylcytosine and 5-methylcytosine from normal to tumor tissues are associated with carcinogenesis and prognosis in colorectal cancer. J Zhejiang Univ Sci B. 2017;18:747-56.

38. Hsu CH, Peng KL, Kang ML, Chen YR, Yang YC, Tsai CH, et al. TET1 Suppresses cancer invasion by activating the tissue inhibitors of metalloproteinases. Cell Rep. 2012;2:568-79.

39. Dong W, Li H, Zhang Y, Yang H, Guo M, Li L, et al. Matrix metalloproteinase 2 promotes cell growth and invasion in colorectal cancer. Acta Biochim Biophys Sin (Shanghai). 2011;43:840-8.
40. Gillison ML, Trotti AM, Harris J, Eisbruch A, Harari PM, Adelstein DJ, et al. Radiotherapy plus cetuximab or cisplatin in human papillomavirus-positive oropharyngeal cancer (NRG Oncology RTOG 1016): a randomised, multicentre, non-inferiority trial. Lancet. 2019;393:40-50.

41. Koneva LA, Zhang Y, Virani S, Hall PB, McHugh JB, Chepeha DB, et al. HPV integration in HNSCC correlates with survival outcomes, immune response signatures, and candidate drivers. Mol Cancer Res. 2017;16:90-102.

42. Cooper T, Biron VL, Adam B, Klimowicz AC, Puttagunta L, Seikaly H. Association of keratinization with 5-year disease-specific survival in oropharyngeal squamous cell carcinoma. JAMA Otolaryngol - Head Neck Surg. 2015;141:250-6.

43. Locati LD, Serafini MS, lannò MF, Carenzo A, Orlandi E, Resteghin C, et al. Mining of self-organizing map gene-expression portraits reveals prognostic stratification of HPV-positive head and neck squamous cell carcinoma. Cancers (Basel). 2019;11:1057.

44. Mohanta S, Siddappa G, Valiyaveedan SG, Ramanjanappa RDT, Das D, Pandian R, et al. Cancer stem cell markers in patterning differentiation and in prognosis of oral squamous cell carcinoma. Tumor Biol. 2017:39:1010428317703656.

45. Rocca A, Farolfi A, Bravaccini S, Schirone A, Amadori D. Palbociclib (PD 0332991): Targeting the cell cycle machinery in breast cancer. Expert Opin Pharmacother. 2014;15:407-20.

46. Zainal NS, Lee BKB, Wong ZW, Chin IS, Yee PS, Gan CP, et al. Effects of palbociclib in oral squamous cell carcinoma and the role of PIK3CA in conferring resistance. Cancer Biol Med. 2019;16:264.

47. Pavlasova G, Mraz M. The regulation and function of CD20: An "enigma" of B-cell biology and targeted therapy. Haematologica. 2020;105:1494-506.

48. Lin TS. Ofatumumab: a novel monoclonal anti-CD20 antibody. Pharmacogenomics Pers Med. 2010;3:51.

49. Colacino JA, Dolinoy DC, Duffy SA, Sartor MA, Chepeha DB, Bradford $C R$, et al. Comprehensive analysis of DNA methylation in head and neck squamous cell carcinoma indicates differences by survival and clinicopathologic characteristics. PLoS ONE. 2013;8:e54742.

50. Bhattacharyya S, Pradhan K, Campbell N, Mazdo J, Vasantkumar A, Maqbool $\mathrm{S}$, et al. Altered hydroxymethylation is seen at regulatory regions in pancreatic cancer and regulates oncogenic pathways. Genome Res. 2017;27:1830-42.

51. Ernst J, Kellis M. ChromHMM: Automating chromatin-state discovery and characterization. Nat Methods. 2012;9:215-6.

52. Cavalcante RG, Patil S, Park Y, Rozek LS, Sartor MA. Integrating DNA methylation and hydroxymethylation data with the mint pipeline. Cancer Res. 2017;77:e27-30.

53. Andrews S, Krueger F, Seconds-Pichon A, Biggins F, Wingett S. FastQC: a quality control tool for high throughput sequence data. Babraham Bioinformatics. Babraham Institute. 2015.

54. Langmead B, Salzberg SL. Fast gapped-read alignment with Bowtie 2. Nat Methods. 2012;9:357.

55. Zhang Y, Lin YH, Johnson TD, Rozek LS, Sartor MA. PePr: A peak-calling prioritization pipeline to identify consistent or differential peaks from replicated ChIP-Seq data. Bioinformatics. 2014;30(18):2568-75.

56. Cavalcante RG, Sartor MA. Annotatr: Genomic regions in context. Bioinformatics. 2017:33(15):2381-3.

57. Heinz S, Benner C, Spann N, Bertolino E, Lin YC, Laslo P, et al. Simple combinations of lineage-determining transcription factors prime cisregulatory elements required for macrophage and $\mathrm{B}$ cell identities. Mol Cell. 2010;38(4):576-89.

58. Tian Y, Morris TJ, Webster AP, Yang Z, Beck S, Feber A, et al. ChAMP: Updated methylation analysis pipeline for Illumina BeadChips. Bioinformatics. 2017:33:3982-4.

\section{Publisher's Note}

Springer Nature remains neutral with regard to jurisdictional claims in published maps and institutional affiliations. 